\title{
Spontaneous Curvature of Phosphatidic Acid and Lysophosphatidic Acid ${ }^{\dagger}$
}

\author{
Edgar E. Kooijman,,$»$ Vladimir Chupin, ${ }^{\ddagger}$ Nola L. Fuller, ${ }^{\S, \nabla}$ Michael M. Kozlov," Ben de Kruijff, \\ Koert N. J. Burger, ${ }^{\perp}$ and Peter R. Rand ${ }^{\S}$ \\ Department of Biochemistry of Membranes, Center for Biomembranes and Lipid Enzymology, Utrecht University, \\ Padualaan 8, 3584 CH Utrecht, The Netherlands, Biological Sciences, Brock University, St. Catherines, Ontario, Canada, \\ Department of Physiology and Pharmacology, Sackler Faculty of Medicine, Tel Aviv University, Tel Aviv, Israel, and \\ Department of Molecular Cell Biology, Institute of Biomembranes, Utrecht University, Utrecht, The Netherlands
}

Received October 6, 2004; Revised Manuscript Received November 16, 2004

\begin{abstract}
The formation of phosphatidic acid (PA) from lysophosphatidic acid (LPA), diacylglycerol, or phosphatidylcholine plays a key role in the regulation of intracellular membrane fission events, but the underlying molecular mechanism has not been resolved. A likely possibility is that PA affects local membrane curvature facilitating membrane bending and fission. To examine this possibility, we determined the spontaneous radius of curvature $\left(R_{0 \mathrm{p}}\right)$ of PA and LPA, carrying oleoyl fatty acids, using well-established $\mathrm{X}$-ray diffraction methods. We found that, under physiological conditions of $\mathrm{pH}$ and salt concentration $(\mathrm{pH} 7.0,150 \mathrm{mM} \mathrm{NaCl})$, the $R_{0 \mathrm{p}}$ values of PA and LPA were $-46 \AA$ and $+20 \AA$, respectively. Thus PA has considerable negative spontaneous curvature while LPA has the most positive spontaneous curvature of any membrane lipid measured to date. The further addition of $\mathrm{Ca}^{2+}$ did not significantly affect lipid spontaneous curvature; however, omitting $\mathrm{NaCl}$ from the hydration buffer greatly reduced the spontaneous curvature of PA, turning it into a cylindrically shaped lipid molecule $\left(R_{0 \mathrm{p}}\right.$ of $\left.-1.3 \times 10^{2} \AA\right)$. Our quantitative data on the spontaneous radius of curvature of PA and LPA at a physiological $\mathrm{pH}$ and salt concentration will be instrumental in developing future models of biomembrane fission.
\end{abstract}

Intracellular membrane transport occurs through the selective fission and fusion of membrane bound transport carriers (TCs), ${ }^{1}$ which contain specific lipid and protein cargo (l). Just prior to fission the TCs consist of a bud, which can be a vesicular or large saccular-tubular (pleiomorphic) structure, connected to the membrane by a narrow neck. Fission is the process that severs the neck releasing the TC from the donor membrane, and fusion is the process by which the TC merges with an acceptor membrane. The molecular rearrangements that accompany fission and fusion are related, both requiring restructuring of the equilibrium bilayer configuration into highly curved non-bilayer intermediates $(2,3)$.

Fission and fusion are highly regulated events and do not occur spontaneously in vivo, but under strict control of

\footnotetext{
† B.d.K., E.E.K., and K.N.J.B. are supported by NWO/FOM/ALW, "Fysische Biologie" program. M.M.K. and K.N.J.B. are supported by the Human Frontier Science Program Organization, and K.N.J.B. is suppported by an EC network grant, HPRN-CT-2002-00259. N.L.F. and P.R.R. are supported by the Natural Sciences and Engineering Research Council of Canada.

* To whom correspondence should be addressed. Tel: +3130253 5511. Fax: +31 30253 3969. E-mail: e.e.kooijman@chem.uu.nl.

Dept. Biochemistry of Membranes, Utrecht University.

$\S$ Brock University.

${ }^{\nabla}$ Currently at McMaster University.

"Tel Aviv University.

${ }^{\perp}$ Dept. Molecular Cell Biology, Utrecht University.

${ }^{1}$ Abbreviations: ARF, ADP-ribosylation factor; BARS, brefeldin A ribosylated substrate; DAG, diacylglycerol; DOPA, dioleoylphosphatidic acid; DOPE, dioleoylphosphatidylethanolamine; DOPS, dioleoylphosphatidylserine; LPA, lysophosphatidic acid; LPAAT, LPA acyl transferase; LPC, lysophosphatidylcholine; PA, phosphatidic acid; PC, phosphatidylcholine; PLD, phospholipase D; TC, transport carrier.
}

specialized proteins. Recent evidence suggests that these proteins do not act alone but in concert with specific membrane lipids, such as phosphoinositides (4-6), diacylglycerol (DAG) $(7,8)$, and phosphatidic acid (PA) $(7,9-11)$. The role of these lipids maybe 2-fold: (i) to recruit specialized proteins to the sites of fission and fusion and (ii) to facilitate membrane bending and the formation of highly curved intermediates, and thus reduce the energy barriers of fission and fusion. In the current study we focus on PA and the related lipid lysophosphatidic acid (LPA).

Formation of PA is a recurring theme in signaling pathways regulating Golgi membrane transport $(10,11)$. In the Golgi, PA is generated by ARF-activated PLD, which converts phosphatidylcholine (PC) into PA and choline (10), by DAG-kinases (7), and by $50 \mathrm{kDa}$ brefeldin A ribosylated substrate (BARS). In in vitro experiments on isolated Golgi membranes, BARS was shown to act as an enzyme converting LPA into PA using long chain acyl-CoA's as the acyl chain donor, and LPA acyltransferase (LPAAT) activity and Golgi membrane fission appeared to be linked (9). Interestingly, endophilin-A1, a protein involved in endocytic membrane fission, has been independently shown to display the same enzymatic activity (12), and the N-terminal lipidmodifying domain of endophilin B1 was very recently shown to be involved in the maintenance of mitochondrial morphology (13). Together these findings suggest that the local formation of PA from LPA, or through any of the aforementioned lipid metabolic pathways, could be part of a universal "lipid machinery" for membrane fission. If such a mechanism exists, it is likely to depend on special biophysical properties of PA. 
Of particular interest is the spontaneous curvature of PA and LPA because local changes in spontaneous monolayer curvature are known to affect membrane fission and fusion events (3, 14-16). Lipid spontaneous (or "intrinsic") curvature is a quantitative measure of the effective lipid molecular shape, and describes the tendency of a lipid species to form non-bilayer (curved) structures (17-20). The aim of the current study was to directly measure the spontaneous curvature (the inverse of the spontaneous radius of curvature) of PA and LPA at a physiological $\mathrm{pH}$ and salt concentration. Our study extends previous more qualitative studies on the molecular shape of (L)PA $(21-24)$, and now allows theoretical models of biomembrane fission to be developed $(3,16)$. We show that, under physiological conditions of $\mathrm{pH}$ and ionic strength, PA has a negative spontaneous curvature that is slightly less pronounced than that of dioleoylphosphatidylethanolamine (DOPE), while LPA has a spontaneous curvature that is considerably more positive than that of lysophosphatidylcholine (LPC).

\section{MATERIALS AND METHODS}

Sample Preparation. 1,2-Dioleoyl-sn-glycero-3-phosphoethanolamine (DOPE), 1,2-dioleoyl-sn-glycero-3-phosphate (monosodium salt) (DOPA), and 1-oleoyl-sn-glycero-3phosphate (sodium salt) (LPA) were purchased from Avanti Polar Lipids (Birmingham, AL). Lipid purity was checked by thin-layer chromatography and judged to be more than 99\%. $n$-Tetradecane (td) was purchased from Sigma-Aldrich Canada Ltd. (Oakville, ON). Water used in the X-ray experiments was doubly distilled.

Lipid stocks, prepared on the basis of dry weight, were dissolved in chloroform/methanol ( $2 / 1$ by volume). In the case of LPA a small ( $\sim 0.6 \%$ of total solvent volume) amount of water was added to fully dissolve the lipid. From these solutions mixtures of different DOPA/DOPE and LPA/DOPE composition were prepared and then dried by rotary evaporation and vacuum desiccation. To relieve interstitial packing stresses, $16 \mathrm{wt} \%$ tetradecane was added to the dried lipid stocks and equilibrated for $72 \mathrm{~h}$ as previously described (25, 26). Aliquots from each stock were hydrated by either (1) adding weighed amounts of water; (2) adding excess amounts of buffer, $25 \mathrm{mM}$ Tes, $25 \mathrm{mM}$ Mes, $150 \mathrm{mM} \mathrm{NaCl}$ set to pH 7.0 using $\mathrm{NaOH}$; or (3) adding excess amounts of buffer as above containing an additional $25 \mathrm{mM} \mathrm{CaCl}_{2}$. Teflon shavings, to provide an internal $\mathrm{X}$-ray calibration standard with a repeat spacing of $4.87 \AA$, were added just prior to sealing the samples between Mylar windows $1 \mathrm{~mm}$ apart.

$X$-ray Diffraction. The hexagonal $\left(\mathrm{H}_{\mathrm{II}}\right)$ phases formed by the various DOPE/DOPA/td and DOPE/LPA/td mixtures were analyzed using X-ray diffraction, as described (2528). A Rigaku rotating anode X-ray generator produced a $\mathrm{Cu} \mathrm{K} \alpha_{1}$ line $(\lambda=1.540 \AA)$, isolated using a bent quartz crystal monochromator. Guinier X-ray cameras were used to capture the diffraction patterns photographically. Samples were investigated at $22.0 \pm 0.5^{\circ} \mathrm{C}$ and hexagonal repeat spacings determined with a measuring error of $\pm 0.1 \AA$. $\mathrm{X}$-ray data analysis was performed according to refs $26-$ 30 .

\section{RESULTS}

Spontaneous Radius of Curvature of DOPA and LPA. In this study we focused on PA and LPA carrying oleoyl
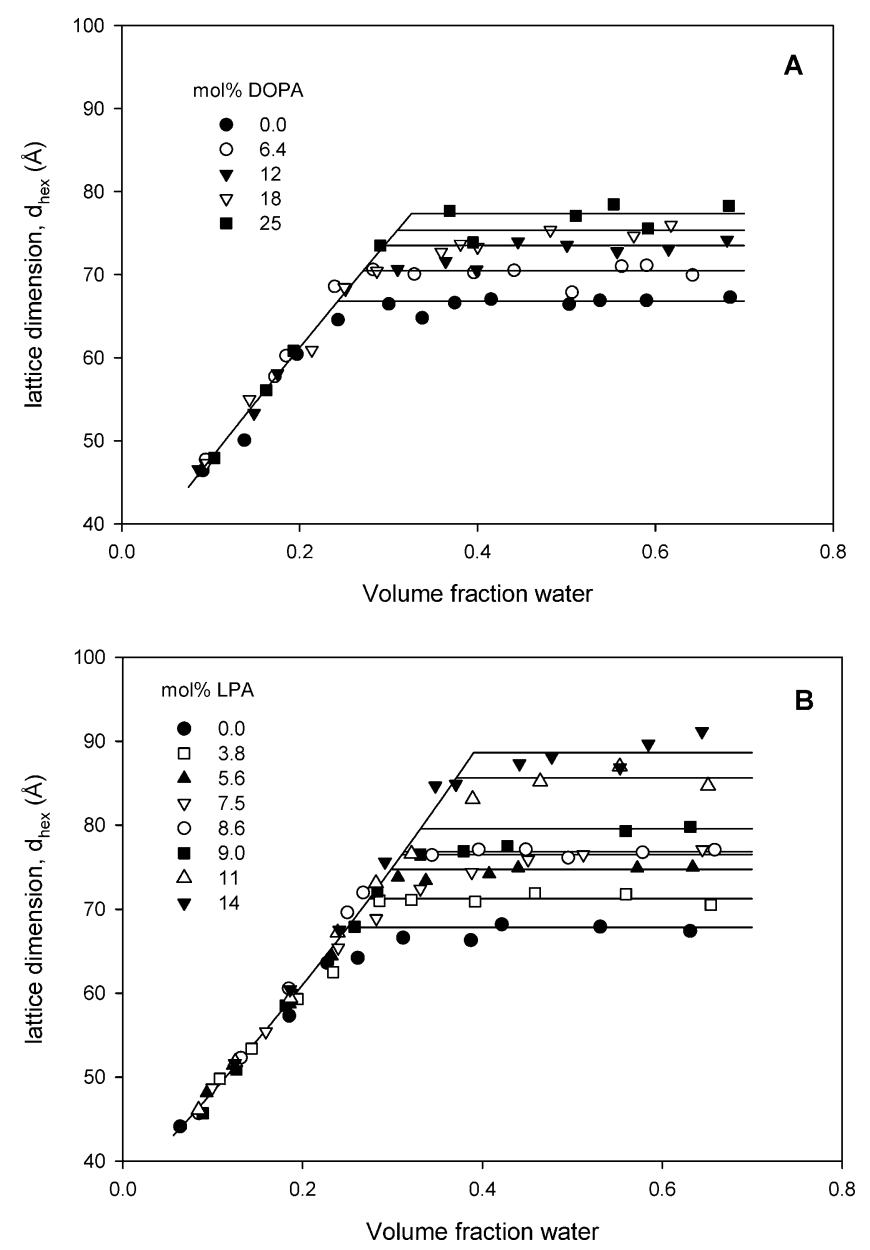

FIGURE 1: Hydration curves relating the hexagonal phase lattice dimension to water content for various mixtures of (A) DOPA/ DOPE/td and (B) LPA/DOPE/td. Horizontal lines represent the equilibrium lattice dimension determined by the average $d_{\text {hex }}$ measured in excess water.

(18:1c) fatty acids, because membrane fission induced by the LPA acyltransferases, BARS and endophilin, appears to be most efficient in the presence of unsaturated fatty acylCoA $(9,12)$. The spontaneous radius of curvature of DOPA and LPA can be determined from the (de)hydration behavior of mixtures containing increasing amounts of (L)PA in DOPE. A prerequisite is that hydrocarbon chain stress is relieved by the addition of 16 wt $\%$ tetradecane $(20,31)$. Under these conditions the curvature that is expressed by the lipid monolayers in the $\mathrm{H}_{\mathrm{II}}$ phase is very close to the spontaneous (intrinsic) curvature, and can be calculated from the hexagonal phase dimension $d_{\text {hex }}$ determined by smallangle X-ray reflection.

Figure 1A and Figure 1B show hydration curves for lipid mixtures of varying composition consisting of DOPA/DOPE/ td and LPA/DOPE/td, respectively. The hexagonal phase dimension $d_{\text {hex }}$ changed with the volume fraction of added water. At low hydration, $d_{\text {hex }}$ increased proportionally with the volume fraction of water. This proportional dependence was used to determine one of the parameters used in the calculation of the spontaneous radius of curvature, namely, the volume fraction of water inside the $\mathrm{H}_{\text {II }}$ phase at full hydration (i.e. in excess water $(20,27))$. A second parameter used in the calculation is the lattice dimension in excess water, the equilibrium lattice dimension, indicated by the horizontal lines in Figure 1A,B. These equilibrium lattice 
Table 1: The Spontaneous Radius of Curvature $R_{0 \mathrm{p}}$ and Spontaneous Curvature $1 / R_{0 \mathrm{p}}$ for $(\mathrm{L}) \mathrm{PA}$ and DOPE in Comparison to Literature Data

\begin{tabular}{|c|c|c|c|c|c|c|c|}
\hline & \multicolumn{2}{|c|}{ water } & \multicolumn{2}{|c|}{ 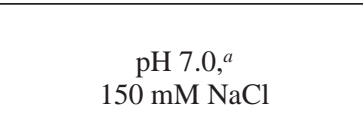 } & \multicolumn{2}{|c|}{$\begin{array}{c}\mathrm{pH} 7.0,^{b} \\
150 \mathrm{mM} \mathrm{NaCl}+ \\
25 \mathrm{mM} \mathrm{Ca}^{2+}\end{array}$} & \multirow[b]{2}{*}{ refs } \\
\hline & $R_{0 \mathrm{p}}(\AA)$ & $1 / R_{0 \mathrm{p}}\left(\AA^{-1}\right)$ & $R_{0 \mathrm{p}}(\AA)$ & $1 / R_{0 \mathrm{p}}\left(\AA^{-1}\right)$ & $R_{0 \mathrm{p}}(\AA)$ & $1 / R_{0 \mathrm{p}}\left(\AA^{-1}\right)$ & \\
\hline $\begin{array}{l}\text { DOPA } \\
\text { LPA }^{c} \\
\text { DOPE }\end{array}$ & $\begin{array}{l}-130 \\
+20 \\
-27 \pm 1^{d}\end{array}$ & $\begin{array}{l}-0.0077 \\
+0.050 \\
-0.037\end{array}$ & $\begin{array}{l}-46 \\
+20 \\
-23 \pm 1^{d}\end{array}$ & $\begin{array}{l}-0.022 \\
+0.050 \\
-0.043\end{array}$ & $\begin{array}{l}-43 \\
+23 \\
-22 \pm 1^{d}\end{array}$ & $\begin{array}{l}-0.023 \\
+0.043 \\
-0.045\end{array}$ & $\begin{array}{l}\text { this study } \\
\text { this study } \\
\text { this study }\end{array}$ \\
\hline $\begin{array}{l}\text { DOPE } \\
\text { DAG }^{c} \\
\text { LPC }^{c} \\
\text { DOPS } \\
\text { DOPC }\end{array}$ & $\begin{array}{l}-28.5 \pm 2 \\
-11 \\
+38 \\
+144 \\
-140 \text { to }-200\end{array}$ & $\begin{array}{l}-0.035 \\
-0.090 \\
+0.026 \\
+0.0069 \\
-0.0071 \text { to } \\
-0.0050\end{array}$ & & & & & $\begin{array}{l}27 \\
27 \\
29 \\
30 \\
26\end{array}$ \\
\hline
\end{tabular}

${ }^{a}$ In buffer $(25 \mathrm{mM}$ Tes, $25 \mathrm{mM}$ Mes set with $\mathrm{NaOH})$ at $\mathrm{pH} 7.0$ containing $150 \mathrm{mM} \mathrm{NaCl} .{ }^{b}$ In buffer $(25 \mathrm{mM}$ Tes, $25 \mathrm{mM} \mathrm{Mes} \mathrm{set} \mathrm{with} \mathrm{NaOH})$ at pH 7.0 containing $150 \mathrm{mM} \mathrm{NaCl}$ and $25 \mathrm{mM} \mathrm{CaCl}_{2} .{ }^{c}$ Oleoyl fatty acid chain(s). ${ }^{d}$ Range between data from PA and LPA datasets $(n=2)$.

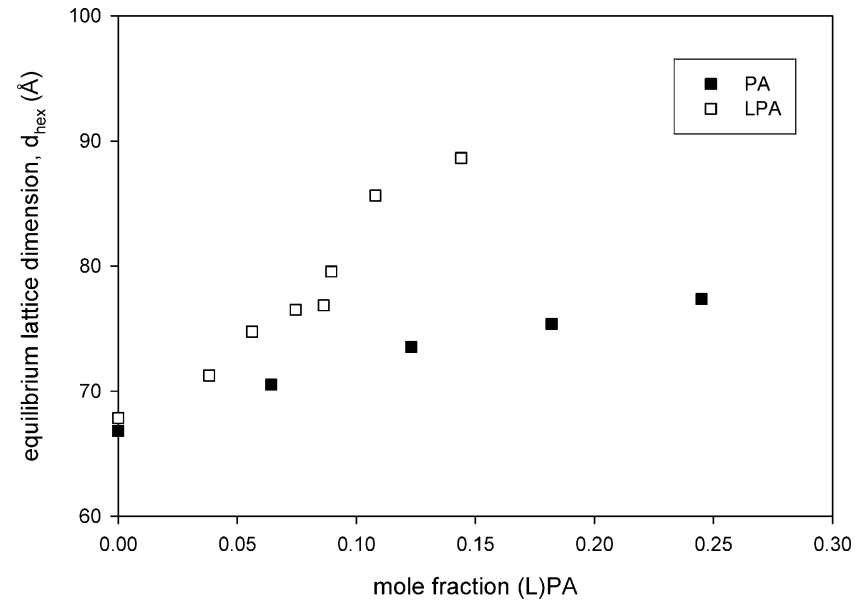

FIGURE 2: Plot of the average equilibrium lattice dimension for DOPA/DOPE/td and LPA/DOPE/td mixtures.

dimensions were obtained by averaging the lattice dimensions at high volume fractions of water, and are shown in Figure 2 as a function of (L)PA content.

To calculate the spontaneous radius of curvature of (L)PA we established that a well-defined pivotal plane exists using so-called diagnostic plots (27). The linearity of these plots (data not shown, linear regression coefficients $r^{2}$ of 0.85 for DOPA and $r^{2}$ of 0.91 for LPA containing mixtures) indicated that for both DOPA and LPA containing samples a pivotal plane could be defined and that the spontaneous curvature, $1 / R_{0 \mathrm{p}}$, to this plane could be calculated. The spontaneous curvature is shown in Figure 3 as a function of (L)PA content. The linear relationship allows the spontaneous radius of curvature of the individual lipids to be calculated (see ref 27). The results are shown in Table 1 and are compared to values previously measured for other lipids.

Effect of Salts on the Spontaneous Curvature of (L)PA. In the X-ray experiments discussed above, samples were hydrated with doubly distilled water and $\mathrm{pH}$ was not controlled. Because screening of lipid headgroup charge by cations is expected to affect lipid packing and spontaneous curvature, we determined the effects of physiological salt concentrations on the lipid spontaneous curvature of (L)PA. Mixtures of DOPA/DOPE/td and LPA/DOPE/td were equilibrated in an excess of buffer containing $150 \mathrm{mM} \mathrm{NaCl}$ at $\mathrm{pH}$ 7.0. The equilibrium lattice spacings were significantly smaller than those found in the samples hydrated in excess
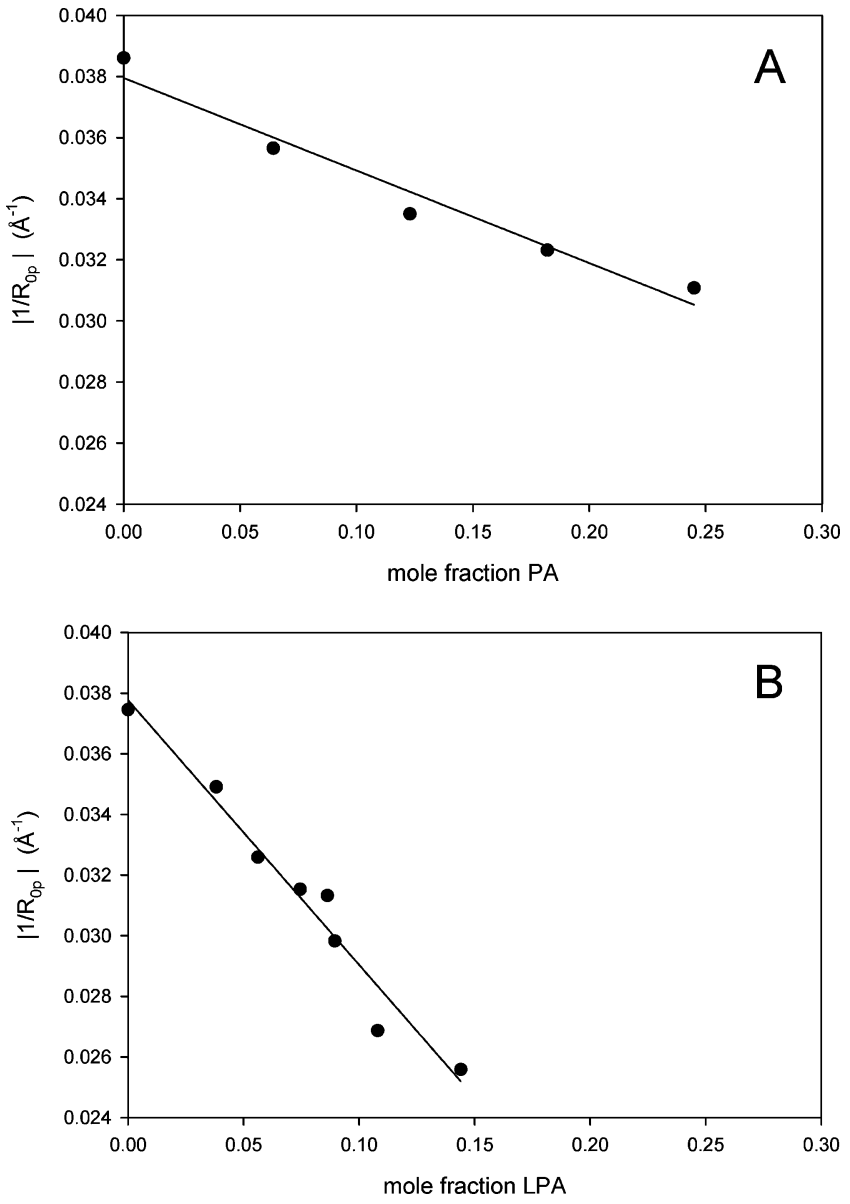

FIGURE 3: Plot of the absolute value of the spontaneous curvature, $\left|1 / R_{0 \mathrm{p}}\right|$, calculated at the pivotal plane for (A) DOPA/DOPE/td and (B) LPA/DOPE/td mixtures as a function of DOPA and LPA content, respectively. Linear regression analysis (in panel $\mathrm{A}, r^{2}=$ 0.96; in panel B, $r^{2}=0.96$ ) gives (A) $R_{0 \mathrm{p}} \mathrm{DOPA}=-1.3 \times 10^{2} \AA$ and $R_{0 \mathrm{p}}$ DOPE $=-26 \AA$ and $(\mathrm{B}) R_{0 \mathrm{p}}^{\mathrm{LPA}}=+20 \AA$ and $R_{0 \mathrm{p}}$ DOPE $=$ $-27 \AA$.

water without $\mathrm{NaCl}$ (Figure $4 \mathrm{~A}, \mathrm{~B}$ ), indicating that the addition of salt results in a more negative spontaneous curvature $\left(1 / R_{0 \mathrm{p}}\right)$ of the mixed lipid monolayers in the $\mathrm{H}_{\mathrm{II}}$ phase. Note that the addition of salt also slightly affected the spontaneous curvature of DOPE (Figure 4 and Table 1) in agreement with published calorimetric data $(32,33)$.

To estimate $R_{0 \mathrm{p}}$ for (L)PA from the equilibrium lattice dimension, $d_{\text {hex }}$, found at $\mathrm{pH} 7.0$ and $150 \mathrm{mM} \mathrm{NaCl}$, we used the proportional dependence found for the hydration behavior 

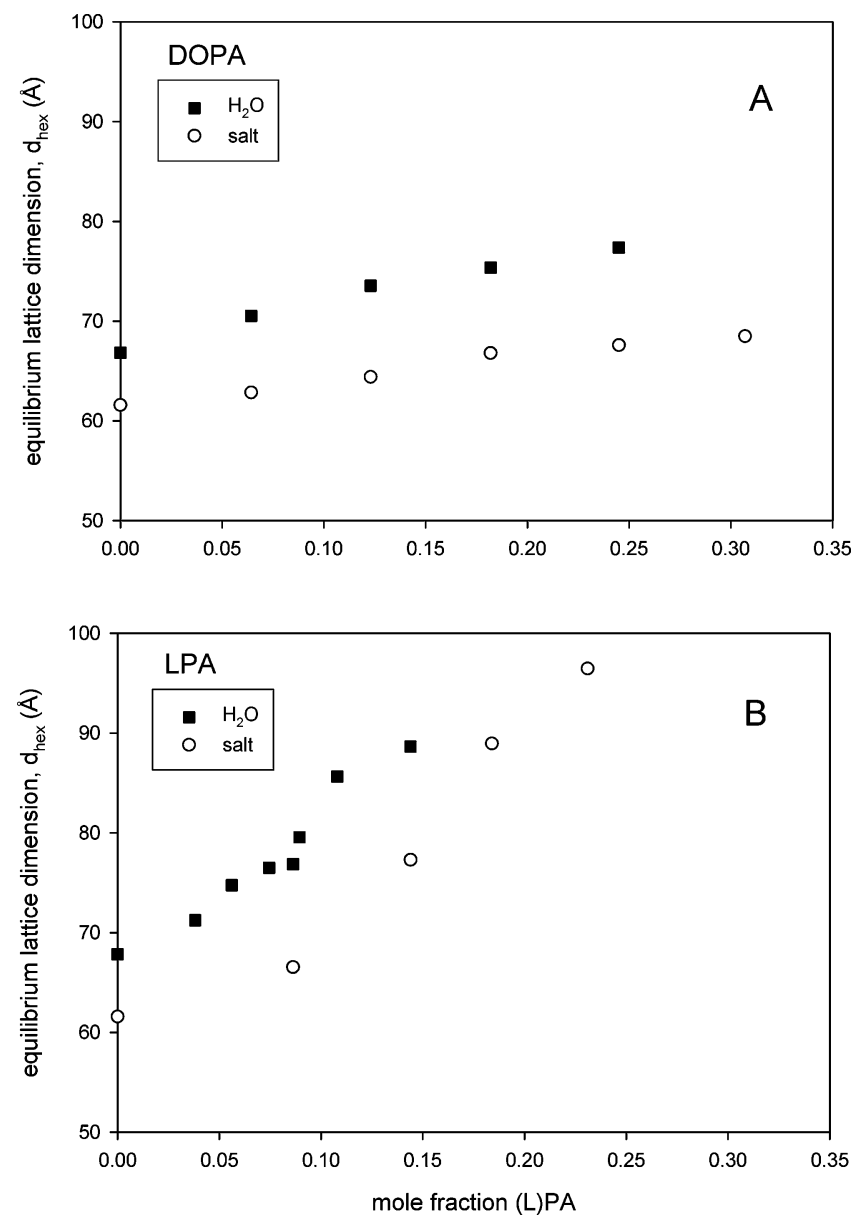

FIGURE 4: Plot of the equilibrium lattice dimension measured in doubly distilled water (squares) and $\mathrm{Mes} / \mathrm{Tes}-\mathrm{NaOH}$ buffer at $\mathrm{pH}$ 7.0 containing $150 \mathrm{mM}$ salt (circles) for (A) DOPA/DOPE/td mixtures and (B) LPA/DOPE/td mixtures.

Table 2: Equilibrium Lattice Parameters, $d_{\text {hex }}$ and $d_{1}$, for DOPA/ DOPE/td Mixtures

\begin{tabular}{|c|c|c|c|c|c|c|}
\hline \multirow{2}{*}{$\begin{array}{l}\text { mol \% } \\
\text { DOPA }\end{array}$} & \multicolumn{2}{|c|}{ water } & \multicolumn{2}{|c|}{$\begin{array}{c}\mathrm{pH} 7.0+ \\
150 \mathrm{mM} \mathrm{NaCl}\end{array}$} & \multicolumn{2}{|c|}{$\begin{array}{c}\mathrm{pH} 7.0+ \\
150 \mathrm{mM} \mathrm{NaCl}+ \\
25 \mathrm{mM} \mathrm{CaCl}_{2}\end{array}$} \\
\hline & $d_{\text {hex }}(\AA)$ & $d_{1}(\AA)$ & $d_{\text {hex }}(\AA)$ & $d_{1}(\AA)$ & $d_{\text {hex }}(\AA)$ & $d_{1}(\AA)$ \\
\hline 0 & 66.8 & 37.3 & 61.6 & 37.5 & 60.5 & 37.5 \\
\hline 6.4 & & 37.0 & 62.8 & 37.4 & 62.9 & 37.4 \\
\hline 12 & 73.5 & 36.7 & 64.4 & 37.4 & 63.7 & 37.4 \\
\hline 18 & 75.3 & 36.4 & 66.8 & 37.3 & 65.0 & 37.4 \\
\hline 25 & 77.4 & 36.1 & 67.6 & 37.2 & 66.3 & 37.3 \\
\hline 31 & $\mathrm{nd}^{a}$ & nd & 68.5 & 37.2 & 67.7 & 37.2 \\
\hline
\end{tabular}

${ }^{a}$ Not determined.

in water, to determine the volume fraction of water (buffer) present in the $\mathrm{H}_{\text {II }}$ phase. We assumed that the lipid molecular dimension, $d_{1}$ (see ref 27), is equal to that found for the corresponding lattice dimension in Figure 1 (i.e. in the presence of only water). This is a reasonable assumption because many previous measurements (see e.g. ref 30) show that changes in $d_{1}$ are small considering the changes observed in the equilibrium lattice dimensions in this study (Tables 2 and 3). The spontaneous curvatures for the individual mixtures were calculated as before and are shown in Figure 5. From the linearity of this plot we determined the spontaneous radius of curvature for DOPA, LPA, and DOPE. The results summarized in Table 1 indicate that the spontaneous curvature of DOPA is strongly affected by $150 \mathrm{mM}$
Table 3: Equilibrium Lattice Parameters, $d_{\text {hex }}$ and $d_{\mathrm{l}}$, for LPA/ DOPE/td Mixtures

\begin{tabular}{|c|c|c|c|c|c|c|}
\hline \multirow{2}{*}{$\begin{array}{c}\text { mol \% } \\
\text { LPA }\end{array}$} & \multicolumn{2}{|c|}{ water } & \multicolumn{2}{|c|}{$\begin{array}{c}\mathrm{pH} 7.0+ \\
150 \mathrm{mM} \mathrm{NaCl}\end{array}$} & \multicolumn{2}{|c|}{$\begin{array}{c}\mathrm{pH} 7.0+ \\
150 \mathrm{mM} \mathrm{NaCl}^{+}+ \\
25 \mathrm{mM} \mathrm{CaCl}_{2}\end{array}$} \\
\hline & $d_{\text {hex }}(\AA)$ & $d_{1}(\AA)$ & $d_{\text {hex }}(\AA)$ & $d_{1}(\AA)$ & $d_{\text {hex }}(\AA)$ & $d_{1}(\AA)$ \\
\hline 0.0 & 67.8 & 37.3 & 61.6 & 37.4 & 60.5 & 37.4 \\
\hline 3.8 & 71.2 & 37.2 & $\mathrm{nd}^{a}$ & nd & nd & nd \\
\hline 5.6 & 74.8 & 36.9 & nd & nd & nd & nd \\
\hline 7.5 & 76.5 & 36.8 & nd & nd & nd & nd \\
\hline 8.6 & 76.8 & 36.8 & 66.7 & 37.3 & 64.9 & 37.4 \\
\hline 9.0 & 79.6 & 36.5 & nd & nd & nd & nd \\
\hline 11 & 85.6 & 35.8 & nd & nd & nd & nd \\
\hline 14 & 88.6 & 35.4 & 77.4 & 36.7 & 74.2 & 37.0 \\
\hline 18 & nd & nd & 86.7 & 35.7 & 82.7 & 36.2 \\
\hline 23 & nd & nd & 98.3 & 33.7 & 91.7 & 34.9 \\
\hline
\end{tabular}

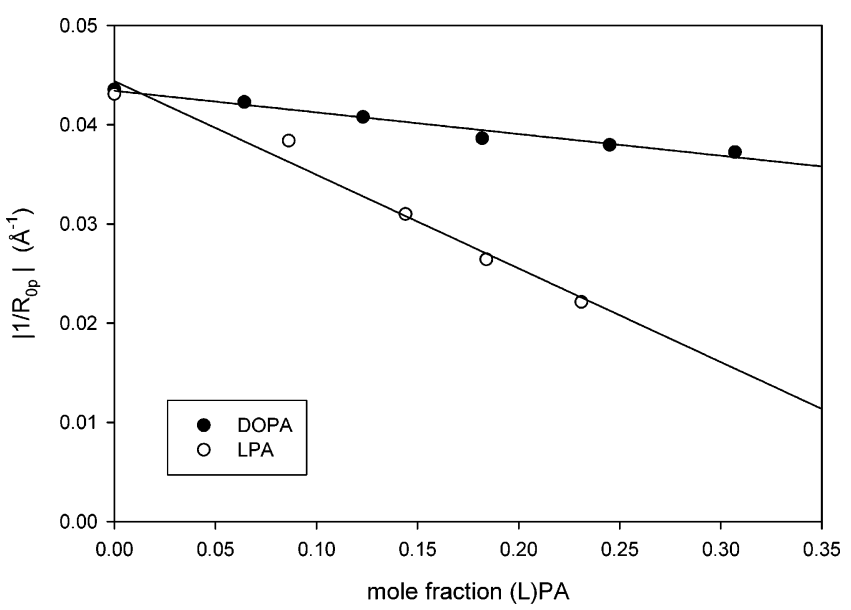

FIGURE 5: Plot of the absolute value of the spontaneous curvature, $\left|1 / R_{0 \mathrm{p}}\right|$, at $\mathrm{pH} 7.0$ in the presence of $150 \mathrm{mM} \mathrm{NaCl}$ calculated at the pivotal plane for DOPA/DOPE/td (solid circles) and LPA/ DOPE/td (open circles) mixtures as a function of DOPA and LPA content, respectively. Linear regression analysis $\left(r^{2}=0.98\right.$ and 0.97 for PA and LPA dataset respectively) gives $R_{0 \mathrm{p}}{ }^{\mathrm{DOPA}}=-46 \AA$, $R_{0 \mathrm{p}}^{\mathrm{LPA}}=+20$, and $R_{0 \mathrm{p}}^{\mathrm{DOPE}}=-22 \pm 1 \AA$.

$\mathrm{NaCl}$, whereas the spontaneous curvature of LPA remains unchanged within experimental error.

Finally we investigated the effect of $\mathrm{Ca}^{2+}$ on the spontaneous radius of curvature in the presence of salt. Samples were equilibrated in buffer at pH 7.0 containing $150 \mathrm{mM} \mathrm{NaCl}$ and $25 \mathrm{mM} \mathrm{CaCl}_{2}$ i.e. in large excess as compared to (L)PA $\left(\mathrm{Ca}^{2+} /(\mathrm{L}) \mathrm{PA}\right.$ molar ratio $\left.\geq 10\right)$. The addition of calcium only slightly (see Tables 2 and 3 ) reduced the equilibrium lattice dimension. The spontaneous radius of curvature calculations (Table 1) indicate that the addition of $\mathrm{Ca}^{2+}$ does not significantly affect the spontaneous curvature of (L)PA at neutral $\mathrm{pH}$ in the presence of physiological salt concentrations.

\section{DISCUSSION}

The local formation of PA plays a key role in the regulation of intracellular membrane fission events, possibly by affecting local membrane curvature. To examine this possibility we determined the spontaneous curvature of DOPA and LPA under physiological conditions of charge and salt concentrations. The spontaneous curvature, $1 / R_{0 \mathrm{p}}$, is the inverse of the spontaneous radius of curvature $R_{0 \mathrm{p}}$. It is a quantitative measure of effective lipid shape, and 
describes the intrinsic propensity of a particular lipid to form curved, non-bilayer, structures $(19,20)$. Table 1 summarizes the spontaneous curvature data obtained for PA and LPA (this study) in comparison to literature data on other membrane lipids. Both the spontaneous radius of curvature $R_{0 \mathrm{p}}$ (first column) and its inverse, the spontaneous curvature $1 / R_{0 \mathrm{p}}$ (second column), are shown. A small (i.e. close to zero) positive or negative spontaneous curvature (i.e. a large positive or negative spontaneous radius of curvature) describes a lipid that prefers a bilayer organization with little intrinsic curvature (e.g. DOPS and DOPC, respectively, see Table 1). Lipids with a large negative spontaneous curvature (a small and negative spontaneous radius of curvature), like DAG, form structures with a large negative monolayer curvature such as $\mathrm{H}_{\text {II }}$ phases, and lipids with a large positive spontaneous curvature, such as LPC, form structures with a large positive monolayer curvature, like spherical micelles or the $\mathrm{H}_{\mathrm{I}}$ phase.

Spontaneous Curvature of DOPA. The spontaneous curvature measured for DOPA in water is close to zero and thus comparable to that of other lipids that form lamellar phases (see Table 1); i.e. under these conditions PA behaves as a cylindrically shaped lipid. Under more physiological conditions of neutral $\mathrm{pH}$ and in the presence of $150 \mathrm{mM}$ salt, PA has a considerably more negative spontaneous curvature, in fact approaching that of DOPE. Further addition of $25 \mathrm{mM} \mathrm{CaCl}_{2}$, at neutral $\mathrm{pH}$, does not have a significant effect on the spontaneous curvature of DOPA.

These results can be understood by considering that lipid spontaneous curvature depends not only on molecular structure but also on intermolecular interactions within the lipid monolayer. Electrostatic repulsion between polar headgroups increases the effective area occupied by the headgroup and, thus, has the effect of adding positive spontaneous curvature. Electrolytes reduce electrostatic repulsion between charged lipid headgroups, decreasing their effective size, and thus shift spontaneous curvature toward more negative values. Note that the change in spontaneous curvature of DOPA induced by salt is not caused by a change in the net charge of the lipid headgroup. We directly determined this charge by solid state ${ }^{31} \mathrm{P}$ NMR methods and found that the headgroup charge on (L)PA in water falls in a relevant physiological range and is not affected by salt addition (data not shown).

The effect of $\mathrm{Ca}^{2+}$ on the spontaneous curvature of DOPA appears to depend critically on lipid headgroup charge. At acidic $\mathrm{pH}$, the average charge of $\mathrm{PA}$ approaches one $\mathrm{e}^{-}$(data not shown) and $\mathrm{Ca}^{2+}$ is expected to bind two PA molecules resulting in an effective lipid molecule with a more negative spontaneous curvature, similar to the effects of $\mathrm{Ca}^{2+}$ binding on the molecular shape of cardiolipin (34). Indeed, submillimolar concentrations of $\mathrm{Ca}^{2+}$ are sufficient to induce a bilayer-to- $\mathrm{H}_{\text {II }}$ phase transition in DOPA at $37^{\circ} \mathrm{C}$, but only at acidic and not at neutral $\mathrm{pH}(24)$. At neutral $\mathrm{pH}, \mathrm{PA}$ carries on average more than one $\mathrm{e}^{-}$(data not shown) and probably does not bind $\mathrm{Ca}^{2+}$ directly. Under these conditions, $\mathrm{Ca}^{2+}$ should merely have a charge screening effect, similar to that mediated by salt, and therefore does not significantly change the spontaneous curvature of PA at physiological salt concentrations. Our finding that, at neutral $\mathrm{pH}$ and in the presence of a physiological salt concentration, $\mathrm{Ca}^{2+}$ does not affect the spontaneous curvature of PA indicates that a local rise in cytosolic $\mathrm{Ca}^{2+}$ concentration is not likely to trigger a change in lipid shape of PA in vivo.

Spontaneous Curvature of $L P A$. The spontaneous curvature of LPA found in water is large and positive. Comparing our results to the literature data (Table 1) we find that LPA has a spontaneous curvature that is larger than that of LPC. Under more physiological conditions of neutral $\mathrm{pH}$ and in the presence of $150 \mathrm{mM}$ salt, LPA appears to have the same spontaneous curvature as in pure water and further addition of $25 \mathrm{mM} \mathrm{CaCl} 2$, at neutral $\mathrm{pH}$, had no significant effect on the spontaneous curvature.

The fact that the spontaneous curvature of LPA in water is larger than that of LPC is remarkable in view of the small size of the phosphate headgroup as compared to the phosphocholine headgroup of LPC. Clearly, the spontaneous curvature of LPA is dominated by lipid headgroup repulsion, which dramatically increases the effective size of the lipid headgroup, similar to the situation described earlier for DOPA in water (see above).

In the case of DOPA, salt addition changed lipid spontaneous curvature from close to zero, i.e. essentially cylindrical, to large and negative. The reasons why salts apparently do not change the spontaneous curvature of LPA are not easily understood but may lie, to a first approximation, in a different effect of salt addition on the effective size of the lipid headgroup, and cross-sectional area of the acyl chain(s). In the case of DOPA, the cross-sectional area of the acyl chains is larger than the effective size of the phosphate headgroup, and, both in the absence and in the presence of salts, lipid packing is dominated by the acyl chains: salts reduce the effective size of the lipid headgroup without major effects on lipid packing or cross-sectional area of the acyl chains. A different situation arises for LPA which carries a single acyl chain, and where lipid packing is dominated by the effective size of the lipid headgroup: salts reduce not only the effective size of the lipid headgroup but also lipid packing. Thus a speculative explanation for the absence of an effect of salts on the spontaneous curvature of LPA assumes that salts reduce both the effective size of the lipid headgroup and the cross-sectional area of the acyl chain, and both to the same extent. However, alternative explanations can as yet not be excluded.

Biological Implications. The conversion of LPA into PA by the fission proteins BARS and endophilin has been implicated in two different membrane fission events, BARS at the level of the Golgi and endophilin in endocytosis. Our data are compatible with the initial hypothesis (12) that a conversion of LPA into PA might impose negative curvature on the membrane facilitating membrane constriction. How this putative change in curvature would facilitate the overall fission process, however, is unclear, and alternative models cannot be excluded (for a comprehensive discussion of alternative models see refs 2 and 24). For example, acylation of LPA not only changes lipid spontaneous curvature but also results in a slight increase in lipid molecular area which might, in principle, drive membrane budding by creating surface area asymmetry between the two membrane leaflets (35). However, such a mechanism requires a considerable transmembrane surface area asymmetry, and is therefore unlikely, given the relatively small amount of LPA present in biomembranes (typically below $0.6 \%$ in Golgi membranes (9)) and the fact that acylation only increases lipid molecular 
area by $\sim 45 \%$. Thus, a role of (L)PA in membrane bending and fission is more likely to be related to a change in local spontaneous curvature than to an increase in global transmembrane surface area asymmetry (also see ref 16 ). Indeed, a recent theoretical study shows that if the conversion of LPA into PA is followed by a further metabolism to DAG, a membrane lipid with a more extreme negative spontaneous curvature $(26,27)$ (see Table 1), then the pearling of Golgi membranes upon treatment with BARS can be understood (16). Although the crucial role of BARS in Golgi membrane fission was recently confirmed in in vivo experiments (36), the role of PA and the putative conversion of LPA into PA have not yet been resolved. A likely possibility is that the conversion of LPA into PA is only one step in a series of steps leading up to fission in which the different lipid and protein factors work synergistically as suggested in a recent study of BARS-dependent mitotic Golgi fragmentation (36). The quantitative data on the spontaneous radius of curvature of PA and LPA at physiological $\mathrm{pH}$ and salt concentration presented in the current study will be instrumental in developing future models of biomembrane fission.

\section{ACKNOWLEDGMENT}

We thank Dave Siegel and Dena Mae Kooijman-Agra for helpful discussions and critical reading of the manuscript and Amanda Bradford-Janke for practical assistance.

\section{REFERENCES}

1. Lippincott-Schwartz, J. (2001) The secretory membrane system studied in real-time. Robert Feulgen Prize Lecture, Histochem. Cell Biol. 116, 97-107.

2. Burger, K. N. J. (2000) Greasing membrane fusion and fission machineries, Traffic 1, 605-13.

3. Kozlovsky, Y., and Kozlov, M. M. (2003) Membrane fission: model for intermediate structures, Biophys. J. 85, 85-96.

4. Cremona, O., and De Camilli, P. (2001) Phosphoinositides in membrane traffic at the synapse, J. Cell Sci. 114, 1041-52.

5. Siddhanta, A., Radulescu, A., Stankewich, M. C., Morrow, J. S., and Shields, D. (2003) Fragmentation of the Golgi apparatus. A role for beta III spectrin and synthesis of phosphatidylinositol 4,5bisphosphate, J. Biol. Chem. 278, 1957-65.

6. Godi, A., Di Campli, A., Konstantakopoulos, A., Di Tullio, G., Alessi, D. R., Kular, G. S., Daniele, T., Marra, P., Lucocq, J. M., and De Matteis, M. A. (2004) FAPPs control Golgi-to-cell-surface membrane traffic by binding to ARF and PtdIns(4)P, Nat. Cell Biol. 6, 393-404.

7. Bankaitis, V. A. (2002) Cell biology. Slick recruitment to the Golgi, Science 295, 290-1.

8. Baron, C. L., and Malhotra, V. (2002) Role of diacylglycerol in PKD recruitment to the TGN and protein transport to the plasma membrane, Science 295, 325-8.

9. Weigert, R., Silletta, M. G., Spano, S., Turacchio, G., Cericola, C., Colanzi, A., Senatore, S., Mancini, R., Polishchuk, E. V., Salmona, M., Facchiano, F., Burger, K. N. J., Mironov, A., Luini, A., and Corda, D. (1999) CtBP/BARS induces fission of Golgi membranes by acylating lysophosphatidic acid, Nature 402, 42933.

10. Roth, M. G., Bi, K., Ktistakis, N. T., and Yu, S. (1999) Phospholipase D as an effector for ADP-ribosylation factor in the regulation of vesicular traffic, Chem. Phys. Lipids 98, 141-52.

11. Siddhanta, A., and Shields, D. (1998) Secretory vesicle budding from the trans-Golgi network is mediated by phosphatidic acid levels, J. Biol. Chem. 273, 17995-8.

12. Schmidt, A., Wolde, M., Thiele, C., Fest, W., Kratzin, H., Podtelejnikov, A. V., Witke, W., Huttner, W. B., and Söling, H. D. (1999) Endophilin I mediates synaptic vesicle formation by transfer of arachidonate to lysophosphatidic acid, Nature 401, $133-41$.
13. Karbowski, M., Jeong, S. Y., and Youle, R. J. (2004) Endophilin $\mathrm{B} 1$ is required for the maintenance of mitochondrial morphology, J. Cell Biol. 166, 1027-39.

14. Chernomordik, L. V., and Kozlov, M. M. (2003) Protein-lipid interplay in fusion and fission of biological membranes, Аnпu. Rev. Biochem. 72, 175-207.

15. Kozlovsky, Y., Chernomordik, L. V., and Kozlov, M. M. (2002) Lipid intermediates in membrane fusion: formation, structure, and decay of hemifusion diaphragm, Biophys. J. 83, 2634-51.

16. Shemesh, T., Luini, A., Malhotra, V., Burger, K. N. J., and Kozlov, M. M. (2003) Prefission constriction of Golgi tubular carriers driven by local lipid metabolism: a theoretical model, Biophys. J. 85, 3813-27.

17. Helfrich, W. (1973) Elastic properties of lipid bilayers: theory and possible experiments, Z. Naturforsch. 28C, 693-703.

18. Israelachvili, J. N., Mitchel, D. J., and Ninham, B. W. (1976) Theory of self-assembly of hydrocarbon amphiphiles into micelles and bilayers, J. Chem. Soc., Faraday Trans. 2 72, 1525-68.

19. Gruner, S. M. (1985) Intrinsic curvature hypothesis for biomembrane lipid composition: a role for nonbilayer lipids, Proc. Natl. Acad. Sci. U.S.A. 82, 3665-9.

20. Rand, R. P., Fuller, N. L., Gruner, S. M., and Parsegian, V. A. (1990) Membrane curvature, lipid segregation, and structural transitions for phospholipids under dual-solvent stress, Biochemistry 29, 76-87.

21. Verkleij, A. J., De Maagd, R., Leunissen-Bijvelt, J., and De Kruijff, B. (1982) Divalent cations and chlorpromazine can induce nonbilayer structures in phosphatidic acid-containing model membranes, Biochim. Biophys. Acta 684, 255-62.

22. Farren, S. B., Hope, M. J., and Cullis, P. R. (1983) Polymorphic phase preferences of phosphatidic acid: A $31 \mathrm{P}$ and $2 \mathrm{H}$ NMR study, Biochem. Biophys. Res. Commun. 111, 675-82.

23. Lee, Y. C., Taraschi, T. F., and Janes, N. (1993) Support for the shape concept of lipid structure based on a headgroup volume approach, Biophys. J. 65, 1429-32.

24. Kooijman, E. E., Chupin, V., de Kruijff, B., and Burger, K. N. J. (2003) Modulation of membrane curvature by phosphatidic acid and lysophosphatidic acid, Traffic 4, 162-74.

25. Rand, R. P., and Fuller, N. L. (1994) Structural dimensions and their changes in a reentrant hexagonal-lamellar transition of phospholipids, Biophys. J. 66, 2127-38.

26. Szule, J. A., Fuller, N. L., and Rand, R. P. (2002) The effects of acyl chain length and saturation of diacylglycerols and phosphatidylcholines on membrane monolayer curvature, Biophys. J. 83, 977-84.

27. Leikin, S., Kozlov, M. M., Fuller, N. L., and Rand, R. P. (1996) Measured effects of diacylglycerol on structural and elastic properties of phospholipid membranes, Biophys. J. 71, 2623-32.

28. Chen, Z., and Rand, R. P. (1997) The influence of cholesterol on phospholipid membrane curvature and bending elasticity, Biophys. J. 73, 267-76.

29. Fuller, N., and Rand, R. P. (2001) The influence of lysolipids on the spontaneous curvature and bending elasticity of phospholipid membranes, Biophys. J. 81, 243-54.

30. Fuller, N., Benatti, C. R., and Rand, R. P. (2003) Curvature and bending constants for phosphatidylserine-containing membranes, Biophys. J. 85, 1667-74.

31. Chen, Z., and Rand, R. P. (1998) Comparative study of the effects of several n-alkanes on phospholipid hexagonal phases, Biophys. J. 74, 944-52.

32. Seddon, J. M., Cevc, G., and Marsh, D. (1983) Calorimetric studies of the gel-fluid (L beta-L alpha) and lamellar-inverted hexagonal ( $\mathrm{L}$ alpha-HII) phase transitions in dialkyl- and diacylphosphatidylethanolamines, Biochemistry 22, 1280-9.

33. Seddon, J. M. (1990) Structure of the inverted hexagonal (HII) phase, and non-lamellar phase transitions of lipids, Biochim. Biophys. Acta 1031, 1-69.

34. Rand, R. P., and Sengupta, S. (1972) Cardiolipin forms hexagonal structures with divalent cations, Biochim. Biophys. Acta 255, 48492.

35. Rauch C., and Farge E. (2000) Endocytosis switch controlled by transmembrane osmotic pressure and phospholipid number asymmetry, Biophys. J. 78, 3036-47.

36. Carcedo, C. H., Bonazzi, M., Spano, S., Turacchio, G., Colanzi, A., Luini, A., and Corda, D. (2004) Mitotic Golgi partitioning is driven by the membrane-fissioning protein CtBP3/BARS, Science $305,93-6$

$\mathrm{BI} 0478502$ 